\title{
Designing anisotropy with waveguide subwavelength structures
}

\author{
Robert Halir, Alaine Herrero-Bermello*, José Manuel Luque-González, Alejandro Ortega-Moñux, \\ Gonzalo Wangüemert-Pérez, Aitor V. Velasco*, Jens H. Schmid ${ }^{\dagger}$, Pavel Cheben ${ }^{\dagger}$, Íñigo Molina-Fernández \\ Universidad de Málaga, Dept. de Ingeniería de Comunicaciones, 29010 Málaga, Spain \\ * Institute of Optics, Spanish National Research Council, 28006 Madrid, Spain \\ + National Research Council Canada, Ottawa, Ontario K1A OR6, Canada \\ Tel: (+34) 952137187,e-mail: rhalir@uma.es
}

\begin{abstract}
Silicon sub-wavelength structures have become a versatile design tool for practical, high-performance integrated optical devices, ranging from highly efficient grating couplers to ultra-broadband beam-splitters. Recently, some of the basic anisotropic properties of these structures have been proposed for advance device design. Here we explore these properties in detail, from the underlying physics to emerging applications in on-chip polarization management.
\end{abstract}

Keywords: subwavelength gratings, anisotropy, homogenization, polarization management

\section{INTRODUCTION}

Subwavelength grating (SWG) waveguides are segmented with a period $(\Lambda)$ smaller than the wavelength of light propagating through them [Fig 1(a)], so that diffraction effects are suppressed, and light experiences an equivalent homogenous material. The refractive index of this metamaterial can be varied between the index of the waveguide core and the cladding material by changing the duty cycle $(a / \Lambda)$ of the structure. This ability to engineer the refractive index is exploited in a variety of high performance silicon photonic devices ranging from low loss fiber-to-chip couplers to compact polarization splitters [1,2]. In most of these applications the SWG is often modelled as an isotropic material. It is, however, well known that such periodic structures are inherently anisotropic [3,4]: essentially light experiences different refractive indexes depending on whether it is polarized parallel (along the $x$ or $y$ axis) or perpendicular (along the $z$ axis) to the grating segments. This interesting property has been exploited only recently. In [5], the use of anisotropic waveguide claddings, which can be implemented with SWGs, was proposed to enhance modal confinement. A multimode interference coupler with ultra-broad bandwidth, exploiting the anisotropy of the SWG to control the propagation constants of higher order modes, was demonstrated in [6]. A similar concept was used to achieve polarization splitting in [7]. Here, we explore how SWGs can be modelled as uniaxial crystals, which provides both valuable physical insight and considerably simplifies the electromagnetic simulation of such structures. More importantly, from the point of view of uniaxial crystals, it becomes clear that rotating the segments, as shown in Fig. 1(b), provides direct control over the anisotropy.
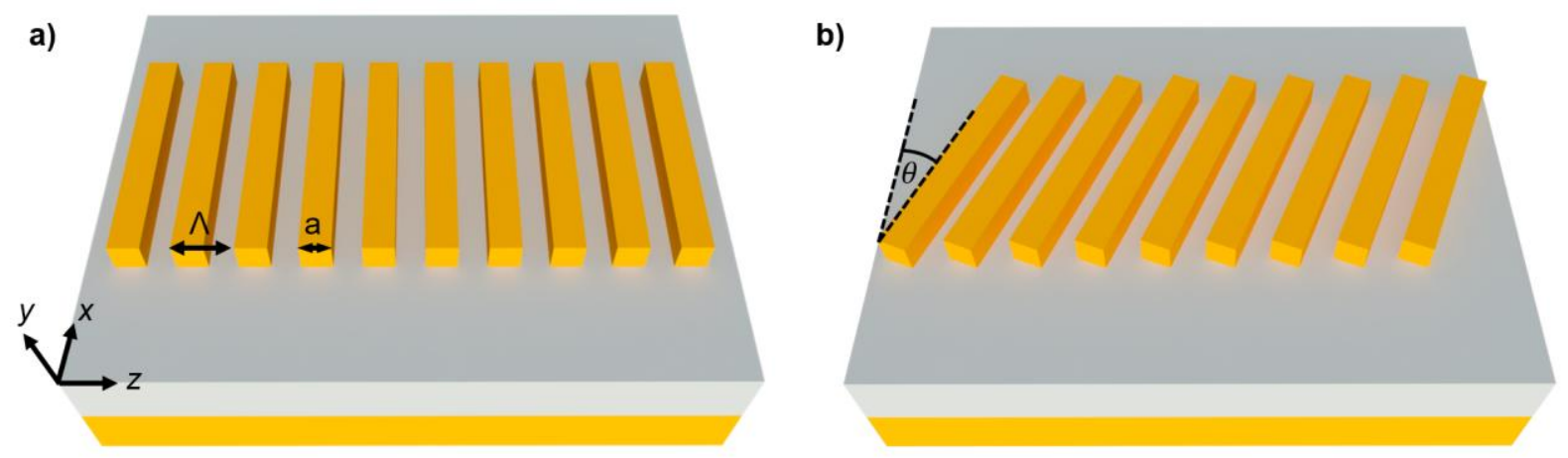

Figure 1. a) In a conventional SWG waveguide light, travelling along the z-direction, experiences a homogenous metamaterial because the pitch of the structure ( 1$)$ is smaller than the wavelength. b) Tilting the waveguide segments with respect to the direction of propagation provides direct control over the anisotropy of the metamaterial.

\section{DISCUSSION}

The dispersion relation of an infinite laminar medium, composed of two periodically alternating materials, is generally rather complex [8], but, for a period smaller than the wavelength, it is well approximated by that of a uniaxial crystal. We postulate that this approximation continues to hold when to both the infinite width and thickness of the laminar medium are shrunk to the finite dimensions typical for SWG waveguides. From the 
dispersion relation in [8], a conventional SWG in silicon on insulator, at a wavelength of $1.55 \mu \mathrm{m}$, with a period of $200 \mathrm{~nm}$ and a $50 \%$ duty cycle, is described by the tensor

$$
n=\left[\begin{array}{ccc}
n_{x x}=2.79 & 0 & 0 \\
0 & n_{y y}=2.79 & 0 \\
0 & 0 & n_{z z}=1.94
\end{array}\right]
$$

To test the accuracy of this approach we study the propagation through a $3 \mu \mathrm{m}$ wide $\mathrm{SWG}$ waveguide $\left(\theta=0^{\circ}\right)$ using both 3D FDTD simulations of the segmented structure, and simple modal analysis of the equivalent uniaxial crystal. As shown in Table 1, the field plots resulting from the 3D FDTD simulations and the model are virtually indistinguishable, for both polarizations, and the relative error in the effective index $\left(n_{\text {eff }}\right)$ is below $3 \%$.

Table 1. Field propagation through SWG waveguides computed with 3D FDTD and modal analysis of the corresponding crystal.

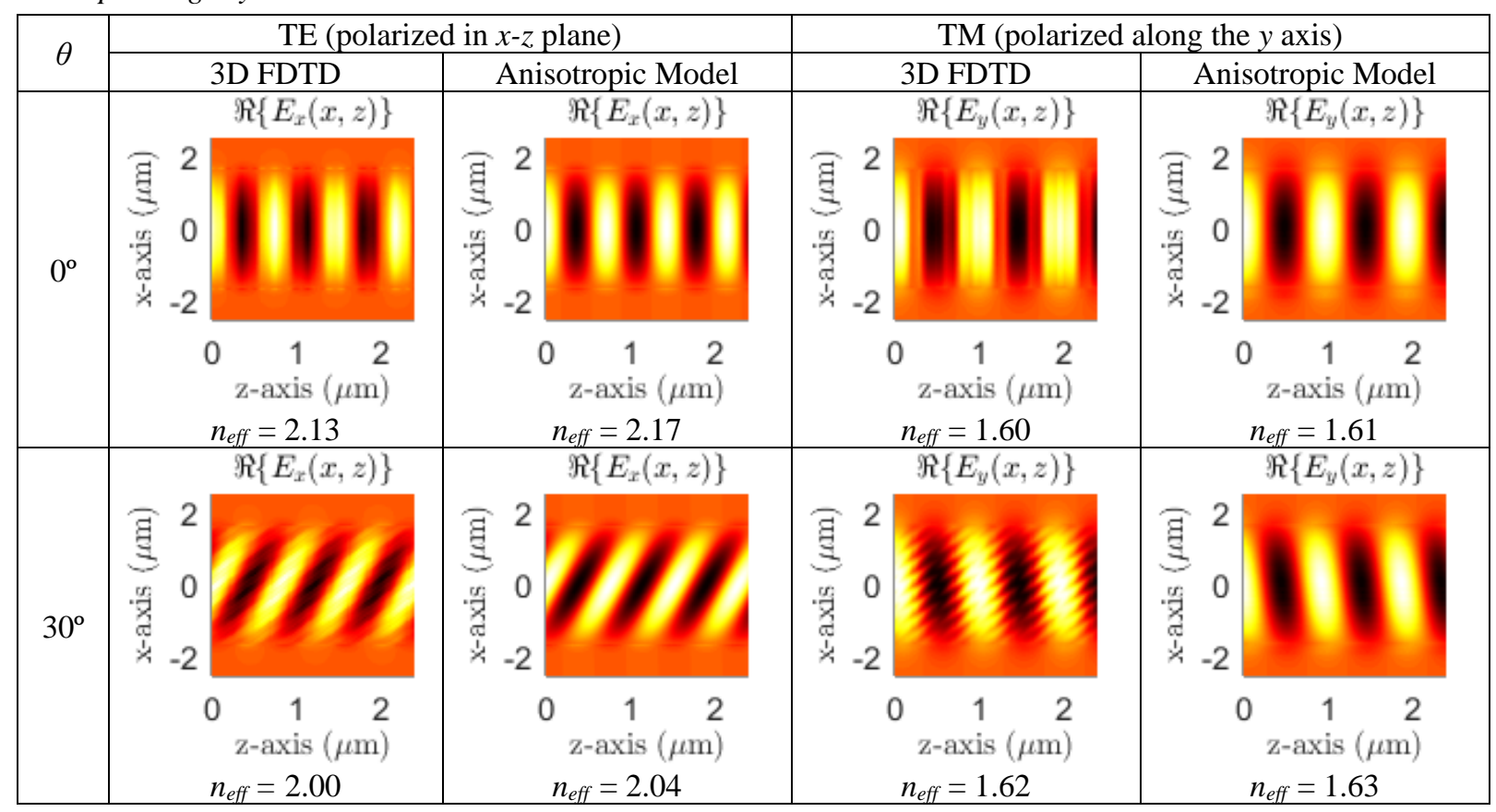

Tilting the SWG segments as shown in Fig. 1(b) is clearly equivalent to a rotation of the crystal, i.e. the tensor in Eq. (1) will be rotated by the angle $\theta$ [9]. Since this rotation leaves the $n_{y y}$ component of the tensor in Eq. (1) constant, one may expect that the TM modes, which are polarized along the $y$ axis, will be mostly unaffected, whereas the TE modes will change. This is observed in the simulations results shown in Table 1 for $\theta=30^{\circ}$, thereby confirming that tilting of the SWG segments provides direct control over the anisotropy of the equivalent metamaterial.

We apply this technique to design the high-performance polarization splitter shown in Fig. 2 . The device is based on a multimode interference coupler, where the beat-length for TE polarization is half the beat-length for TM polarization. As a result, the devices images a TE polarized input from port 1 to the upper output (port 2), whereas a TM polarized input is imaged to the lower output (port 3).
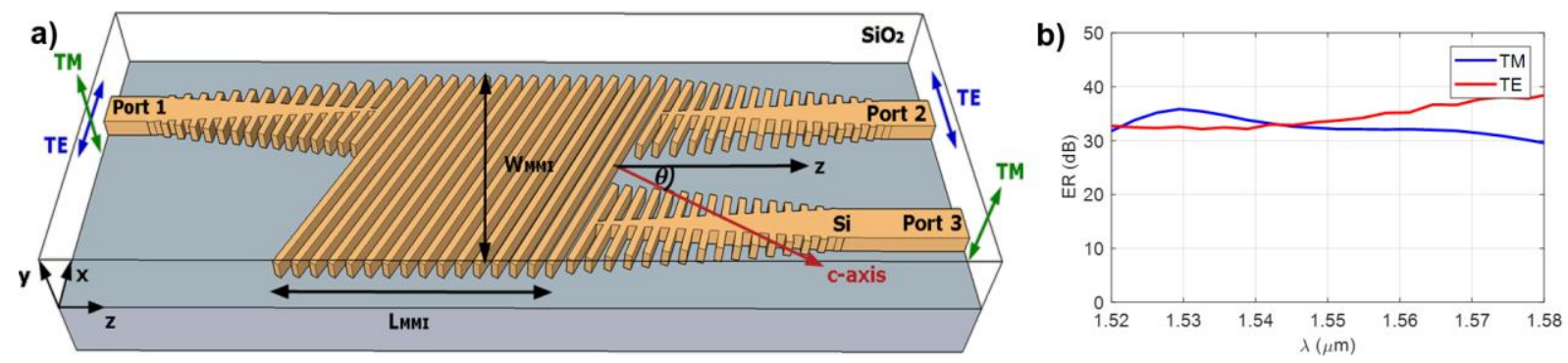

Figure 2. a) By tilting the SWG segments, this multi-mode interference polarization splitter can be independently optimized for TE and TM polarization. b) 3D FDTD simulations of the device reveal virtually wavelength independent extinction ratios, in excess of $30 \mathrm{~dB}$, for both polarizations. 
By using this strategy, we were able to adjust the beat-lengths for both polarizations simply by changing the tilting angle of the structure to $\sim 12^{\circ}$, so the remaining geometric parameters could be used to optimize the performance of the device. As shown in Fig. 2(b), 3D FDTD simulations of the complete structure show that this results in very high extinction ratios for both polarizations, that remain virtually constant over the complete $\mathrm{C}$ band.

\section{CONCLUSIONS}

We have proposed and validated a model, based on uniaxial crystals, that represents the intrinsic anisotropy of SWG waveguides. We have furthermore shown that that tilting the SWG segments provides control over the anisotropy of structures, as predicted by the model. This new degree of freedom can be used to design high performance integrated polarization management devices.

\section{ACKNOWLEDGEMENTS}

We acknowledge funding from the Ministerio de Economía y Competitividad, Programa Estatal de Investigación, Desarrollo e Innovación Orientada a los Retos de la Sociedad (cofinanciado FEDER), Proyectos TEC2016-80718-R, TEC2015-71127-C2-1-R (FPI scholarship BES-2016-077798), and IJCI-2016-30484, the Community of Madrid (S2013/MIT-2790), the Universidad de Málaga, the EMPIR program (JRP-i22 14 IND13 Photind), co-financed by the participating countries and the European Union's 2020 research and innovation program, and the Horizon 2020 research and innovation program under the Marie Sklodowska-Curie grant No. 734331.

\section{REFERENCES}

[1] P. Cheben, P. J. Bock, J. H. Schmid, J. Lapointe, S. Janz, D.-Xia Xu, Adam Densmore, André Delâge, Boris Lamontagne, and Trevor J. Hall: Refractive index engineering with subwavelength gratings for efficient microphotonic couplers and planar waveguide multiplexers, Opt. Lett., vol. 35, pp. 2526-2528, 2010.

[2] R. Halir, P. J. Bock, P. Cheben, A. Ortega-Moñux, C. Alonso-Ramos, J. H. Schmid, J. Lapointe, D.-X. Xu, J. G. Wangüemert-Pérez, Í. Molina-Fernández, et al: Waveguide sub-wavelength structures: a review of principles and applications, Laser Photonics Rev., vol. 9, pp. 25-49, 2015

[3] S. M. Rytov: Electromagnetic properties of a finely stratified medium, Soviet Physics JETP, vol. 2, pp. 466-475, 1956.

[4] D. C. Flanders: Submicrometer periodicity gratings as artificial anisotropic dielectrics, Appl. Phys. Lett., vol. 42, pp. 492-494, 1983.

[5] S. Jahani and Z. Jacob: Breakthroughs in photonics 2014: relaxed total internal reflection, IEEE Photon. J., vol. 7, p. 0700505, 2015.

[6] R. Halir, P. Cheben, J. M. Luque-González, J. Darío Sarmiento-Merenguel, J. H. Schmid, G. WangüemertPérez, D.-X. Xu, S. Wang, A. Ortega-Moñux, and Í. Molina-Fernández: Ultra-broadband nanophotonic beamsplitter using an anisotropic sub-wavelength metamaterial, Laser Photonics Rev., vol. 10, pp. 10391046, 2016.

[7] L. Xu, Y. Wang, A. Kumar, D. Patel, E. El-Fiky, Z. Xing, R. Li, and D. V. Plant: Polarization beam splitter based on mmi coupler with SWG birefringence engineering on SOI, IEEE Photon. Technol. Lett., vol. 30, pp 403-406, 2018.

[8] A. Yariv and P. Yeh: Electromagnetic propagation in periodic stratified media. II. Birefringence, phase mathing, and x-ray lasers, J. Opt. Soc. Am., vol. 67, pp. 438-448, 1977.

[9] D. Marcuse: Modes of a symmetric slab optical waveguide in birefringent media-part I: Optical axis not in plane of slab, IEEE J. Quantum Electron., vol. 14, pp. 736-741, 1978. 